\title{
MAGNETIC ORDER AND ANISOTROPY IN FERRIMAGNETIC CHAINS OF Mn(II) IONS AND NITRONYL-NITROXIDE RADICALS
}

\author{
A. Caneschi $\left({ }^{1}\right)$, D. Gatteschi $\left({ }^{1}\right)$, J. P. Renard $\left({ }^{2}\right)$, P. Rey $\left({ }^{3}\right)$ and R. Sessoli $\left({ }^{1}\right)$ \\ $\left({ }^{1}\right)$ Dipartimento di Chimica, Università di Firenze, Via Maragliano 75/77, 50144, Firenze, Italy \\ $\left(^{2}\right)$ Institut d'Electronique Fondamentale, CNRS UA 22, Bât. 220, Université Paris Sud, 91405, Orsay Cedex, \\ France \\ ( $\left.{ }^{3}\right)$ CENG-DRF, Laboratoire de Chimie, 85X, 38041 Grenoble Cedex, France
}

Abstract. - The magnetic properties of single crystals of Mn (hfac) ${ }_{2}$ NITR with NITR = nitronyl-nitroxide radical with different substituents $R$ have been investigated. The ferrimagnetic chains of alternating Mn II ions and radicals show $3 D$ ferromagnetic order below about $8 \mathrm{~K}$. The anisotropic behaviour is interpreted by taking into account dipolar interactions.

Molecular compounds which exhibit a magnetic transition to a three-dimensional (3D) ordered phase with a large spontaneous magnetization are of current interest. A promising way for obtaining such molecular magnets consists in alternating regularly on a lattice high spins and low spins coupled by antiferromagnetic interaction. Several examples of bimetallic ferrimagnetic chains are known to date $[1,2]$. Some of them with $\mathrm{Mn} \mathrm{II}$ and $\mathrm{Cu}$ II as alternating ions display a ferromagnetic $3 \mathrm{D}$-ordering of the ferrimagnetic chains $[2$, $3]$ and consequently they have a large magnetic moment even in low field, below the critical temperature $T_{\mathrm{c}}$.

We study here the low temperature $3 \mathrm{D}$ magnetic transition of novel compounds with alternating Mn II and free radicals of spins $1 / 2[4,5]$ coupled by a very strong antiferromagnetic interaction: $J \simeq-400 \mathrm{~K}$. Their general formula is $\mathrm{Mn}(\mathrm{hfac})_{2}$ NITR where hfac is hexafluoroacetylacetone, and NITR stable organic radicals, nitronyl-nitroxide with various substituents $\mathrm{R}$. Three different compounds were studied with $\mathrm{R}=$ Ethyl (Et); n-propyl (nPr) and isopropyl (iPr). Mn (hfac) $)_{2}$ NITiPr crystallizes in the monoclinic system with the space group P 2 $/ c$. [4]. The chains of alternating $\mathrm{Mn}$ and NITiPr run along the $c$ axis. The crystal structure of the two other compounds is expected to be likely similar.

Magnetic susceptibility measurements were performed on single crystals along three orthogonal axes including the chain axis, by means of a low field SQUID magnetometer [6] at temperatures down to $2 \mathrm{~K}$. In addition the hysteresis loops were recorded by a conventional fluxmeter method in the $T$ range 1.2-4.2 K.

The susceptibility data versus $T$ for $\mathrm{Mn}$ (hfac) $)_{2}$ NITiPr differ largely for the three axes (Fig. 1). Along the easy axis $b$, the susceptibility displays a plateau below about $7.6 \mathrm{~K}$, whose value is determined by the demagnetizing factor of the sample. This clearly demonstrates $3 \mathrm{D}$ ferromagnetic order between the entities of AF coupled $\mathrm{MnII}$ and radical $(S=2)$. Along

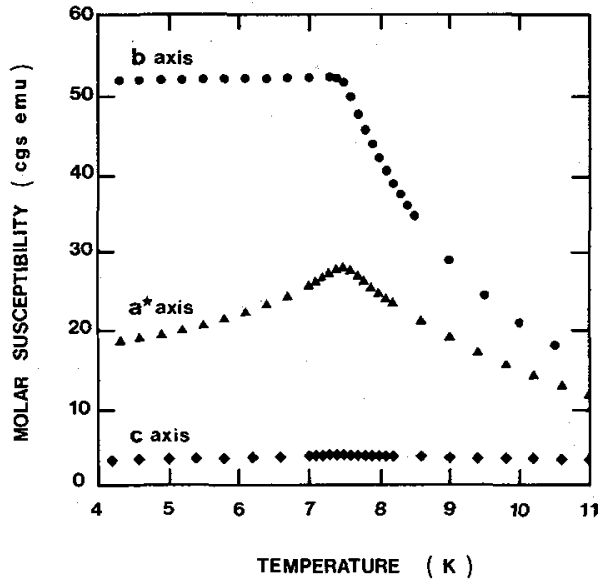

Fig. 1. - Molar susceptibility vs. temperature of $\mathrm{Mn}$ (hfac) NIT iPr in a measuring field of $0.5 \mathrm{Oe}$ along three orthogonal crystal axes.

the hard axis $\mathbf{c}$ and the intermediate axis $\mathbf{a}^{*}$, the susceptibility is much lower, which reflects the existence of not negligible magnetic anisotropy. This latter one was estimated from the susceptibility values extrapolated at $T=0$ and corrected from demagnetizing field. The resulting anisotropy fields acting on the entities of $S=2$ are 1.2 kOe and 7.5 kOe for respectively $\mathrm{a}^{*}$ and c axis. The anisotropy is mainly induced by the dipolar interaction since the single ion anisotropy of $\mathrm{Mn}$ II and the exchange anisotropy between $\mathrm{Mn}$ II and radical are expected to be rather small. In a point dipole approximation, we obtained the dipolar energy for different spin directions: $E_{\mathrm{d}}=-0.139 \mathrm{~K} / / \mathrm{a}^{*} ;-0.126 \mathrm{~K} / / \mathrm{b}$ and $+0.265 \mathrm{~K} / / \mathrm{c}$. This crude model gives $\mathrm{c}$ axis as hard direction and a corresponding anisotropy field of a few kOe roughly consistent with the experiment.

The transition temperature $T_{\mathrm{c}}$ and the critical exponent $\gamma$ of magnetic susceptibility were obtained from accurate measurements performed along $b$ every 
$0.01 \mathrm{~K}$ in the range $7.5-8.1 \mathrm{~K}$. The susceptibility corrected from the demagnetizing field follows the critical law $\chi=\Gamma\left(T-T_{\mathrm{c}}\right)^{-\gamma}$ with $T_{\mathrm{c}}=7.61 \mathrm{~K}$ and $\gamma=1.13$. This abnormally low $\gamma$ value reflects the importance of dipolar interactions in the 3D ordering process. Indeed, $\gamma=1$ for the dipolar uniaxial ferromagnet, while $\gamma=1.38$ for the 3D Heisenberg ferromagnet. An estimation of $T_{\mathrm{c}}$ can be done from the correlation length $\xi=|J| S / 4 k T[7]$ and the strength of dipolar interaction $E_{\mathrm{d}}$. We find $T_{\mathrm{c}} \simeq \sqrt{10^{3} E_{\mathrm{d}}} \simeq 12 \mathrm{~K}$, of the order of magnitude of the experimental value $T_{\mathrm{c}}=7.61 \mathrm{~K}$. The importance of dipolar interactions as major source of magnetic anisotropy is also shown by the electron spin resonance (ESR) spectra. Indeed, low temperature shifts of the ESR field to higher values along $c$ axis, and to lower values for $a^{*}$ and $b$ are observed. The lowest field value corresponds to the easy axis $b$. These results are consistent with the theoretical expectations for ferrimagnetic chains in the framework of the model of Nagata and Tazuke [8].

The two other compounds Mn (hfac) $)_{2}$ NITR with $\mathrm{R}=\mathrm{Et}$ and $\mathrm{nPr}$ show rather similar magnetic behavior with respective $T_{c}$ values of $7.8 \mathrm{~K}$ and $8.3 \mathrm{~K}$. In both compounds, the hard axis is along the chain, but contrarily to $\mathrm{Mn}$ (hfac) ${ }_{2} \mathrm{NiTiPr}$, irreversibility effects occur below $T_{\mathrm{c}}$ in applied field parallel to the eaxy axis. As shown in figure 2, the field cooled (FC) magnetization differs from zero field cooled (ZFC) one obtained by applying the field below $T_{\mathrm{c}}$ on the crystal cooled in zero field. In the same way, an hysteresis loop and a large remnant magnetization are observed below $T_{\mathrm{c}}$. These effects are clearly related to a blocking of the motion of the domain walls which occurs shightly below $T_{c}$. Indeed the ZFC susceptibility along the easy axis, when lowering temperature below $T_{c}$, first exhibits a narrow plateau and then an increase due to the growing up spontaneous magnetization. Far below $T_{c}$, the susceptibility would be proportional to spontaneous magnetization. For Mn (hfac) NITEt, the observed temperature variation between $2 \mathrm{~K}$ and $5 \mathrm{~K}$ is well described by the usual $T^{3 / 2}$ law expected for the low $T$ spontaneous magnetization of a $3 \mathrm{D}$ ferromagnet.

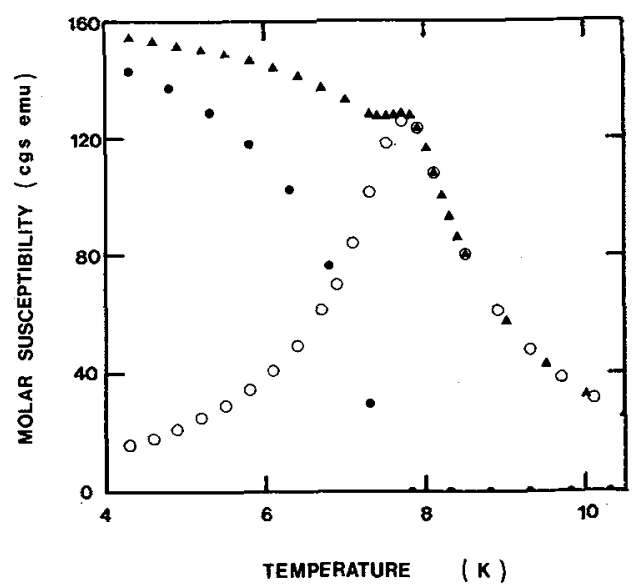

Fig. 2. - Field cooled $(\Delta)$ and zero field cooled ( 0 ) susceptibility, and remnant magnetization divided by the cooling field of $0.5 \mathrm{Oe}(\bullet)$ applied along the easy axis of $\mathrm{Mn}(\mathrm{hfac})_{2}$ NIT Et.

[1] Landee, C. P., Organic and Inorganic LowDimensional Crystalline Materials, Eds. Delhaes P. and Drillon M., NATO ASI Series (Plenum, New York) 68 (1988) 75.

[2] Kahn, O., idem Ref. [1], p. 93.

[3] Kahn, O., Pei, Y., Verdaguer, M., Renard, J. P. and Sletten, J., J. Am. Chem. Soc. 110 (1988) 782.

[4] Caneshi, A., Gatteschi, D., Rey, P. and Sessoli, R., Inorg. Chem. (in press).

[5] Benelli, C., Caneschi, A., Gatteschi, D. and Rey, P., idem Ref. [1], p. 109;

Caneschi, A., Gatteschi, D., Laugier, J., Rey, P., Sessoli, R. and Zanchini, C., idem Ref. [1], p. 381.

[6] Beauvillain, P., Chappert, C. and Renard, J. P., J. Phys. E 18 (1985) 839.

[7] Seiden, J., J. Phys. Lett. 44 (1983) L947.

[8] Nagata, K. and Tazuke, Y., J. Phys. Soc. Jpn 32 (1972) 337. 